ARTICLE

https://doi.org/10.1038/s41467-019-11506-0

\title{
Direct atomic insight into the role of dopants in phase-change materials
}

Min Zhu (1) 1, Wenxiong Song ${ }^{1}$, Philipp M. Konze (1) 2, Tao Li', Baptiste Gault (i) ${ }^{3,4}$, Xin Chen ${ }^{1}$, Jiabin Shen ${ }^{1}$, Shilong Lv' ${ }^{1}$, Zhitang Song ${ }^{1}$, Matthias Wuttig (10) ${ }^{5,6}$ \& Richard Dronskowski (i) ${ }^{2,7,8}$

Doping is indispensable to tailor phase-change materials (PCM) in optical and electronic data storage. Very few experimental studies, however, have provided quantitative information on the distribution of dopants on the atomic-scale. Here, we present atom-resolved images of $\mathrm{Ag}$ and In dopants in $\mathrm{Sb}_{2} \mathrm{Te}$-based (AIST) PCM using electron microscopy and atom-probe tomography. Combing these with DFT calculations and chemical-bonding analysis, we unambiguously determine the dopants' role upon recrystallization. Composition profiles corroborate the substitution of $\mathrm{Sb}$ by $\mathrm{In}$ and $\mathrm{Ag}$, and the segregation of excessive $\mathrm{Ag}$ into grain boundaries. While $\mathrm{In}$ is bonded covalently to neighboring $\mathrm{Te}, \mathrm{Ag}$ binds ionically. Moreover, In doping accelerates the crystallization and hence operation while Ag doping limits the random diffusion of In atoms and enhances the thermal stability of the amorphous phase.

\footnotetext{
${ }^{1}$ State Key Laboratory of Functional Materials for Informatics, Shanghai Institute of Micro-System and Information Technology, Chinese Academy of Sciences, 200050 Shanghai, China. ${ }^{2}$ Chair of Solid-State and Quantum Chemistry, Institute of Inorganic Chemistry, RWTH Aachen University, 52056 Aachen, Germany. ${ }^{3}$ Max-Planck-Institut für Eisenforschung GmbH, 40237 Düsseldorf, Germany. ${ }^{4}$ Department of materials, Imperial College London, London SW7 2AZ, UK. ${ }^{5}$ I. Institute of Physics (IA), RWTH Aachen University, 52056 Aachen, Germany. ${ }^{6}$ Peter Grünberg Institute (PGI 10), 
O ver the last 50 years, computers have revolutionized almost every aspect of modern life, in particular communication, education, entertainment, and science, too. Today, however, they face increasing demands for faster data access and larger storage capacity, which are both severely limited by the presently available memory (fast, volatile, small) and storage (slow, non-volatile, large) hierarchies ${ }^{1,2}$. One successful approach for technological improvement is the introduction of phase-change memory ${ }^{3}$, also marketed by Intel/Micron as $3 \mathrm{D}$ Xpoint ${ }^{4}$. It takes advantage of the ability to switch the resistance upon the phase transition from a disordered amorphous (logic 1) to an ordered crystalline phase (logic 0 ) in certain chalcogenides, providing nonvolatility, nanoseconds speed and $4 \mathrm{~F}^{2}$ (F: feature size) density ${ }^{5,6}$, thereby bridging the performance gap between memory and storage ${ }^{3}$. The successful commercialization of phasechange memory has been enabled by successful doping chalcogenides in the pseudo-binary $\mathrm{GeTe}-\mathrm{Sb}_{2} \mathrm{Te}_{3}$ family ${ }^{7,8}$, as well as $\mathrm{Ag}$ and In doping $\mathrm{Sb}_{2}$ Te alloy abbreviated as AIST, in which between 3.4 at. $\%$ and 11 at. $\% \mathrm{Ag}$ and In have been incorporated ${ }^{9,10}$. Thanks to enhanced erasability and sensitivity, AIST has been frequently used in rewritable optical-storage media such as CDRW and DVD-RW, already since $1993^{9,10}$. Moreover, AIST is capable of recrystallizing on the nanosecond time scale from an amorphous-crystalline rim. This enables growth-dominated recrystallization behavior ${ }^{11,12}$, which offers increased potential for DRAM/SRAM-like phase-change memory applications.

Until now, however, very few experimental studies have provided quantitative information on the role of the $\mathrm{Ag}$ and In dopants in AIST, due to limits of analytical technology. This hinders further material optimizations to boost device performance, especially with regards to operation speed and device lifetime. In the earliest report from 1993, Iwasaki et al. employed $\mathrm{X}$-ray diffraction (XRD) and transmission electron microscopy (TEM) and showed that AIST, with 11 at.\% Ag and In, crystallized not as a single phase but as a solid mixture of $\operatorname{AgInTe}_{2}$ and $\mathrm{Sb}$ phases after annealing at $230^{\circ} \mathrm{C}^{10}$. They also observed a solidstate reaction of these phases to yield $\mathrm{AgSbTe}_{2}$ and $\mathrm{InSb}$ as the temperature increases to $350^{\circ} \mathrm{C}$. In 2001, Matsunaga et al. utilized synchrotron-radiation XRD and proposed a uniform A7 crystal structure $(R \overline{3} m)$ with just one crystallographic site, randomly occupied by all atoms (Ag, In, $\mathrm{Sb}$, or $\mathrm{Te}$ ). It subsequently changes upon annealing to a rhombohedral structure at $\sim 507^{\circ} \mathrm{C}^{13}$. In contrast, Raoux et al. discovered that the XRD pattern of AIST resembled pure $\mathrm{Sb}_{2} \mathrm{Te}$, characterized by the repeated stacking of $\mathrm{Sb}_{2}$ bilayers and $\mathrm{Sb}_{2} \mathrm{Te}_{3}$ quintuple layers ${ }^{14}$. Based on a statistical technique called fluctuation TEM, Lee et al. directly detected the nanometer-scale nuclei embedded in AIST and their evolution over time ${ }^{15}$. Later, Matsunaga et al. combined extended X-ray absorption fine structure (EXAFS), hard X-ray photoelectron spectroscopy (HXPS) and reverse Monte Carlo (RMC) simulations, and determined a distortedly octahedral atomic arrangement of $\mathrm{Sb}$ and $\mathrm{Te}$ atoms but were less successful to explain the precise role of $\mathrm{Ag}$ and In atoms ${ }^{12}$. In both aforementioned cases, as well as in the recent density-functional theory (DFT) calculations $^{16}$, a randomly mixed structure of AIST was used.

In this contribution, we provide atomic insight of the distribution of 5.13 at.\% $\mathrm{Ag}$ and 3.32 at.\% In within polycrystalline thin films of AIST, the composition of which is close to that used in rewriteable optical-storage media such as DVD-RW ( $\sim$ at.\% $\mathrm{Ag}$ and $\sim 5$ at. $\%$ In $)^{5}$. First their occupancy in the main structure of the grains is revealed by atomically resolved aberrationcorrected scanning TEM (Cs-corrected STEM). In addition, quantitative information is provided by atom-probe tomography (APT) performed specifically at grain boundaries. Ultimately, the structure model obtained is fed into density-functional theory calculations and analyzed by chemical-bonding theory, through which the precise role of each element in the recrystallization process is revealed.

\section{Results}

Structure and chemical identifications. Figure 1 presents the atomic arrangement and element distributions of crystalline AIST obtained from in situ TEM investigation. The atomically resolved high-angle annular dark-field (HAADF) images in Fig. 1a show that crystalline AIST annealed at $200^{\circ} \mathrm{C}$ is stacked by both bilayer and quintuple-layer units. $\mathrm{Ag}$, In, $\mathrm{Sb}$, and $\mathrm{Te}$ are distributed inhomogeneously, with $\mathrm{Ag}$ and In showing a preference to the $\mathrm{Sb}_{2} \mathrm{Te}_{3}$ quintuple layers, while the bilayers are built from mostly Sb. After increasing the temperature to $350{ }^{\circ} \mathrm{C}$ (Fig. $1 \mathrm{~b}-\mathrm{d}$ ), the changes in atomic arrangement are miniscule, while the degree of crystallinity increases with higher heating temperature, as indicated by clearer atomic energy-dispersive X-ray spectroscopy (EDX) mappings.

To investigate the influence of annealing time on the atomic arrangement, the sample was annealed at $300{ }^{\circ} \mathrm{C}$ for $30 \mathrm{~min}$, so Fig. 2 shows additional micrographs after this annealing step. Apparently, as seen from the HAADF image in Fig. 2a, crystalline AIST shows a bilayer stacking sequence, separated by narrow gaps, which is commonly found in crystalline AIST ${ }^{13}$. The observed atomic arrangement remains the same as that observed in Fig. 1, which suggests that the crystalline structure of AIST is barely affected by the annealing time. Correspondingly, the line profile taken across four bilayers shows two kinds of atomic column distances (Supplementary Fig. 1), $2.06 \AA$ and 2.61 $\AA$, in line with the Matsunaga model (Supplementary Fig. 2) ${ }^{13}$. The atomic configuration is identical to that of the rhombohedral (A7-type) $\mathrm{Sb}$ crystal projected along the $<001>$ direction $^{17}$. A $\sim 20 \%$ variation in bond lengths (three shorter and three longer bonds) is observed, which is attributed to the three-dimensional energy-lowering Peierls distortion of the ideal rocksalt structure $^{18}$. These bilayer structures (marked by yellow boxes) are replaced in some regions, as shown in Fig. 2a, by quintuple layer (highlighted by green boxes). Two interplanar distances are shown in the quintuple layer (Supplementary Fig. 1F), namely $2.22 \AA$ and $2.34 \AA$, slightly larger than those of the interlayer distances. The quintuple layer is surrounded by bilayers at a spatial width of $2.48 \AA$. Thereby, AIST has a crystalline structure resembling undoped $\mathrm{Sb}_{2} \mathrm{Te}$, characterized by a systematic stacking of ordered bi- and quintuple-layer blocks (as proposed by Kifune et al. ${ }^{19}$ ), which is significantly different from the repeated bilayer stacking in the Matsunaga model ${ }^{13}$. However, these two building blocks of $\mathrm{Sb}_{2} \mathrm{Te}$ are randomly distributed in crystalline AIST (comparing Fig. 2 and Supplementary Fig. 1), lacking the distinct order found in the binary compound.

While the intensity of the atoms in HAADF images is approximately proportional to $Z^{1.7}$ (ref. ${ }^{20}$ ), suggesting a Te-Sb$\mathrm{Te}-\mathrm{Sb}-\mathrm{Te}$ quintuple layer, the figures still lack information regarding Ag and In. This is attributed to the presence of four elements with similar atomic numbers and low Ag and In concentrations. Further atom-resolved EDX was used to resolve the occupancy, as shown in Fig. $2 b$ to h. Apparently, as seen from Fig. 2a, two quintuple blocks are separated by five continuously stacked bilayers. Indeed, the stacking sequence of a quintuple layer is Te1-Sb-Te2-Sb-Te1, as mentioned above, which can be observed directly from the $\mathrm{Sb}$, Te and their overlay mappings in Fig. 2d to f, respectively. Noticeably, as shown in Fig. 2b, c, the atomically resolved signals from $\mathrm{Ag}$ and In atoms stem from the same sites within the quintuple layers as the $\mathrm{Sb}$ atoms. These dopants thus substitute $\mathrm{Sb}$, and are hence dubbed $\mathrm{Ag}_{\mathrm{Sb}}$ and $\mathrm{In}_{\mathrm{Sb}}$ with the $\mathrm{Sb}$ subscript denoting the substitution site. They are more visualized in the overlaid Ag + Te and $\mathrm{In}+\mathrm{Te}$ in Fig. $2 \mathrm{~g}, \mathrm{~h}$, 

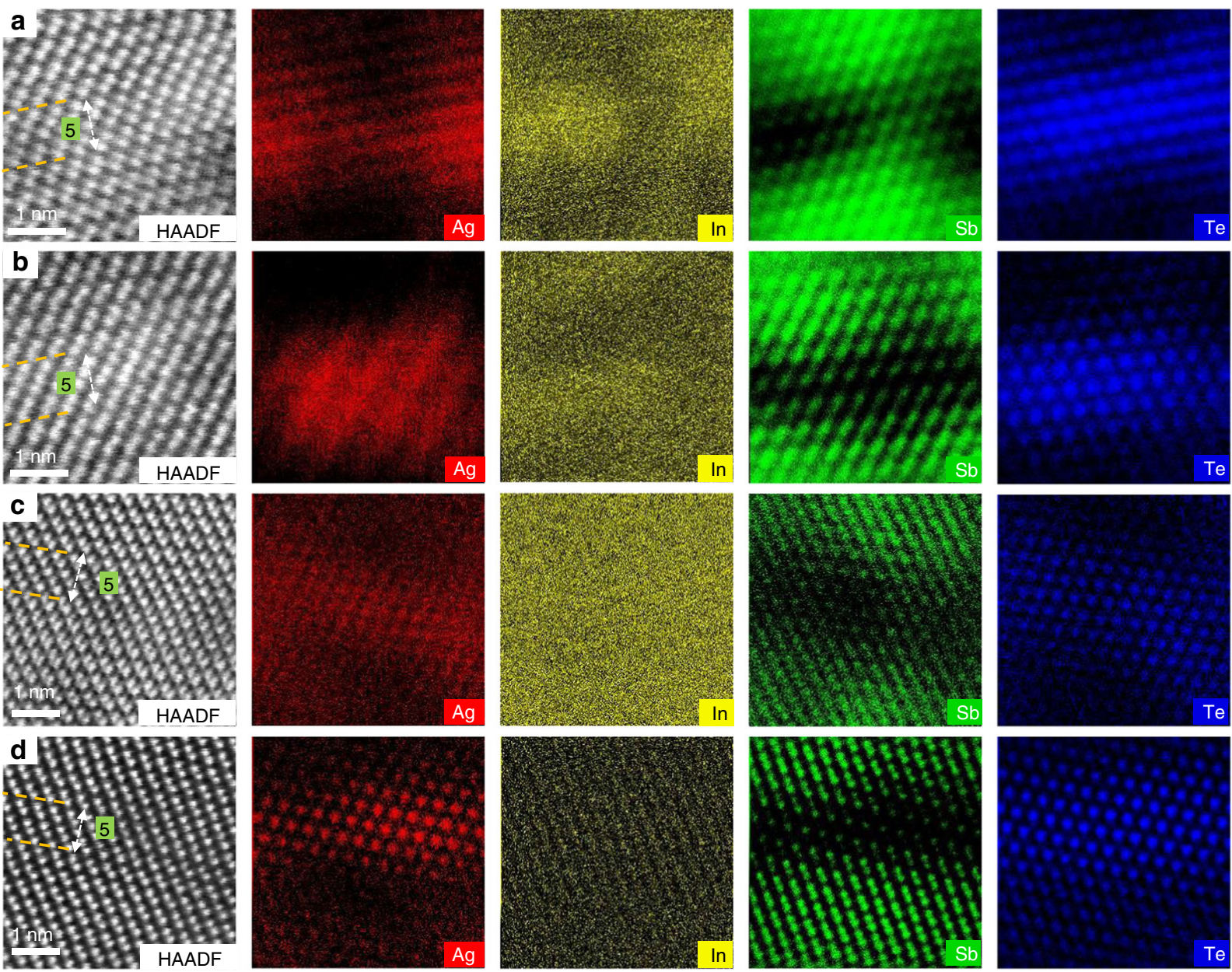

Fig. 1 Atomic structure of crystalline AIST annealed at different temperatures. a-d HAADF-STEM images, and corresponding EDX mappings of Ag, In, Sb and Te elements annealed at $200,250,300$, and $350^{\circ} \mathrm{C}$, respectively. The scale bar is $1 \mathrm{~nm}$

respectively. In contrast, $\mathrm{Sb}$ exclusively occupies the bilayers between the Te-Sb-Te-Sb-Te quintuple layers, and no significant amount of Te, Ag, or In can be detected inside the bilayers. As shown in Supplementary Fig. 3, $\mathrm{Ag}_{\mathrm{Sb}}$ and $\mathrm{In}_{\mathrm{Sb}}$ defects are dramatically reduced as a defective quintuple-layer splits into bilayer and triple-layer (with a small gap between them), as also observed in the crystalline Ti-Sb-Te alloy ${ }^{21,22}$. Summarizing, the atomic stacking model of crystalline AIST is schematically presented in Fig. 2i. The layered stacking configuration obviously differs from the randomly mixed model proposed by Matsunaga et al. in $2001^{13}$.

Element distributions in the grain boundary. Noticeably, Ag atoms not only locate in the $\mathrm{Sb}_{2} \mathrm{Te}_{3}$ quintuple layer, forming $\mathrm{Ag}_{\mathrm{Sb}}$ defects, but also aggregate in the grain boundary (GB), as shown in the EDX mappings in Supplementary Fig. 4. In contrast, fewer $\mathrm{In}, \mathrm{Sb}$ and $\mathrm{Te}$ atoms are found in the grain boundary, supported by the lower intensity than those inside the grains. To provide quantitative information on the distribution of $\mathrm{Ag}$ within both grains and GB, we performed correlative TEM-atom-probe tomography investigations. APT has high elemental sensitivity $(>1 \%$ o $)$ and provides sub-nanometer-scale mapping in threedimensions ${ }^{23}$. The principle of APT is illustrated in the method section and highlighted in ref. ${ }^{24}$. Figure 3 A shows the HAADFSTEM image of the needle-shaped APT specimen. The presence of a high-angle GB is assessed by the different diffraction patterns on either side of the feature highlighted by the red arrow.
Figure $3 \mathrm{~B}$ presents the corresponding reconstructed threedimensional (3D) mapping obtained from APT. The location of the grain boundary is indicated by the dark orange dashed arrow. A set of iso-surfaces encompassing regions of the point cloud containing over 7.5 at.\% $\mathrm{Ag}$ and 5.5 at.\% In are overlaid onto the point cloud in red and yellow, respectively. The thresholds were adjusted to highlight the non-homogeneous distribution of these elements throughout the microstructure. The one-dimensional composition profile displayed in Fig. $3 c$ was calculated along a $d=50 \mathrm{~nm}$ cylindrical region-of-interest positioned normal to the grain boundary, as indicated in the inset, in which a top-view of the grain boundary is displayed. The dashed lines correspond to the average composition within the grains. Inside both grains, the composition is 65.5 at.\% Sb, 26.6 at.\% Te, 3.9 at.\% Ag, and 3.3 at. $\%$ In. The grain boundary is In-depleted, with a composition dropping to $\sim 2$ at. $\%$, which contrasts with a statistically significant segregation of $\mathrm{Ag}$ up to 6 at.\%. This result is consistent with the EDX mappings in Supplementary Fig. 4. From the grain boundary, a $40 \mathrm{~nm}$-thick region extends into the grain. This layer is slightly enriched in $\mathrm{Ag}$, to $\sim 5$ at.\%, and in $\mathrm{Te}$ at 27 at.\%, accompanied by a corresponding depletion of $\mathrm{Sb}$ to 63.5 at.\% and of In to 2.8 at.\%. This means that all In atoms substitute Sb atoms, whereas excessive Ag appears segregated at the grain boundary.

An average composition profile for $14 \mathrm{Ag}$-rich particles sitting at the boundary was calculated as a function of the distance to the isosurface (referred to as a proximity histogram ${ }^{25}$ ) and is shown in Fig. 3d. The Ag composition can reach up to over 33 at.\%, indicative of a strong tendency for phase separation. An Ag 

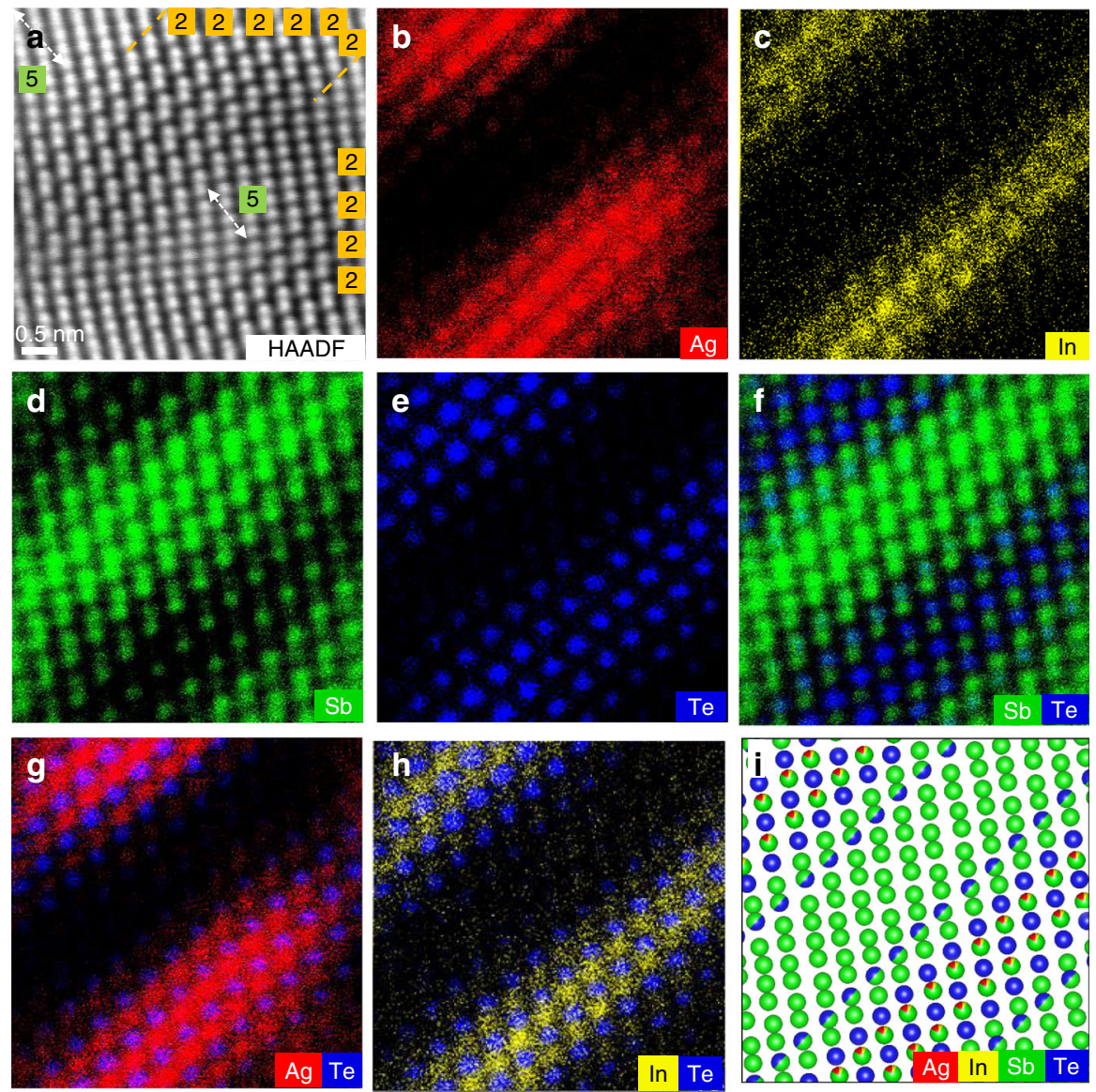

Fig. 2 Structural and chemical identifications of an AIST crystallite, stacked by bilayers and quintuple layers. a HAADF-STEM image; the arrows mark the quintuple layers between two bilayers. b-h EDX mappings for $\mathrm{Ag}$, In, Sb, $\mathrm{Te}, \mathrm{Sb}+\mathrm{Te}, \mathrm{Ag}+\mathrm{Te}$, and In $+\mathrm{Te}$ elements, respectively. I: corresponding schematic atomic stacking model of crystalline AIST. The scale bar is $0.5 \mathrm{~nm}$

particle is also directly observed in a low-angle GB in Supplementary Fig. 5, the core of which is indeed free of $\mathrm{In} / \mathrm{Sb} /$ Te. In addition, In-rich clusters in the range of $5-10 \mathrm{~nm}$ in diameter are observed inside the grains but not at the GB or the interface. The composition profile calculated as a function of the distance to the In-isosurface, shown in Fig. 3e, evidences the coexistence of In and Ag, accompanied by a strong depletion in $\mathrm{Sb}$ and a slight increase in Te. This suggests that these areas are akin to the quintuple layer with $\mathrm{Ag}_{\mathrm{Sb}}$ and $\mathrm{In}_{\mathrm{Sb}}$, as found in the EDX mapping in Fig. 2.

Crystal orbital Hamilton population and chemical-bonding analysis. Through Cs-TEM and APT, we qualitatively determine the chemical and structural arrangement of crystalline AIST as follows: $\mathrm{Sb}$ and Te form Te-Sb-Te-Sb-Te quintuple layers, which are separated by randomly stacked $\mathrm{Sb}$-Sb bilayers; $\sim 3.9$ at.\% Ag and $\sim 3.3$ at.\% In substitute the $\mathrm{Sb}$ in the quintuple layer, forming $\mathrm{Ag}_{\mathrm{Sb}}$ and $\mathrm{In}_{\mathrm{Sb}}$ point defects, whereas excess $\mathrm{Ag}$ (above 3.9 at.\%) separates in or along GBs. Hence, both Cs-corrected and APT results suggest consistently that there are basic units in AIST with different atomic arrangements, different composition, as well as different dopant distribution. These results also explain the phase segregation in AIST with 8-11 at.\% Ag and In, as observed by Iwasaki et al. ${ }^{10}$, while a single rhombohedral phase was found in AIST with only 3.4 at.\% $\mathrm{Ag}$ and 3.7 at.\% In by Matsunaga et al. ${ }^{13}$. No In segregation is found because the maximum solubility of $\mathrm{In}_{\mathrm{Sb}}$ in $\mathrm{Sb}_{2} \mathrm{Te}$ is $\sim 5$ at. $\%{ }^{26}$. To understand why AIST favors this particular structural configuration, the formation energies can be obtained from density-functional theory. The corresponding calculations show that this structure, in which both $\mathrm{Ag}_{\mathrm{Sb}}$ and $\mathrm{In}_{\mathrm{Sb}}$ defects are located in the quintuple layers, is energetically favorable, as displayed in Fig. 4a. More specifically, the total electronic energy of M1 model with both $\mathrm{Ag}_{\mathrm{Sb}}$ and $\mathrm{In}_{\mathrm{Sb}}$ substituents inside the quintuple layer is $-401.18 \mathrm{eV}$, which is $0.71 \mathrm{eV}$ and $0.92 \mathrm{eV}$ lower than those for the M2 (with one In $_{\mathrm{Sb}}$ substituent inside bilayer) and the M3 model (with one $A_{S}$ sb substituent inside bilayer), respectively. We also find that $\mathrm{Ag}$ and In atoms always prefer to be located in the Sb sites of the quintuple layers in AIST as isolated dopants (see Supplementary Fig. 6).

Further projected crystal orbital Hamilton population (pCOHP) bonding analysis ${ }^{27}$ in Fig. $4 \mathrm{~b}$ confirms that the In-Te bonds in model M1 are slightly more stable, by ca. $0.8 \mathrm{eV}$, than the $\mathrm{In}-\mathrm{Sb}$ bonds in $\mathrm{M} 2$, as reflected by the integrated pCOHP values of -12.8 and $-13.6 \mathrm{eV}$. The latter (IpCOHP) values are the bonds' contributions to the band-structure energy (equivalent to the sum of the Kohn-Sham eigenvalues). In this very Fig. 4, spikes to the right denote bonding, and those to the left indicate antibonding levels ${ }^{28}$. While this is in line with the total energy of the system, the trend is not reproduced for $\mathrm{Ag}-\mathrm{Te}$ and $\mathrm{Ag}-\mathrm{Sb}$ interactions because the latter come out stronger by $0.5 \mathrm{eV}$, suggesting Ag cannot be stabilized through interactions of covalent character, which COHP analysis mirrors. To address this, additional Mulliken charge analysis was performed also using LOBSTER ${ }^{29,30}$. The results of this analysis are summarized in Table 1, where we observe a strong difference between the two dopants. While In carries very little charge (and a slightly negative one, so behaving like a mild anion), Ag behaves as a typical 
a

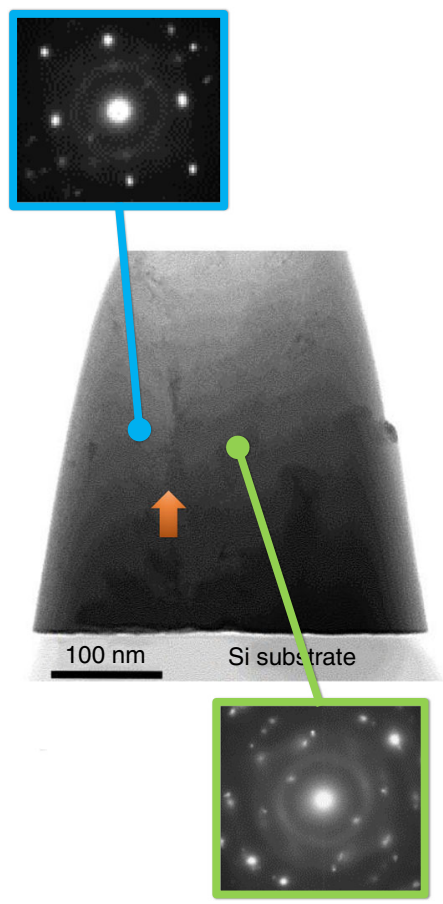

b

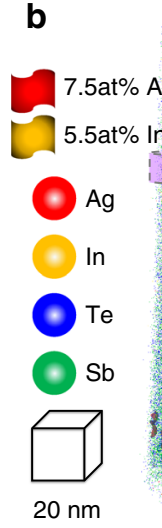

d $^{70}$

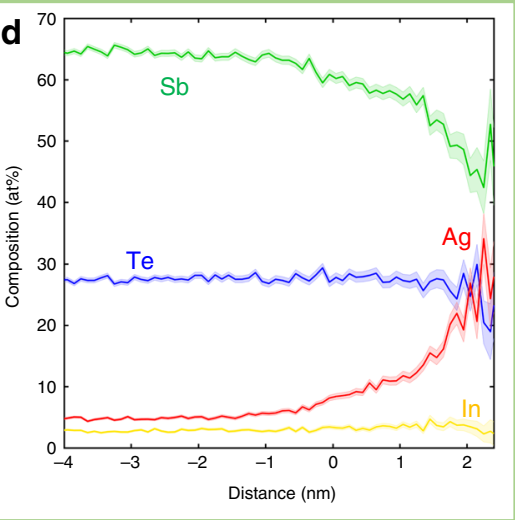

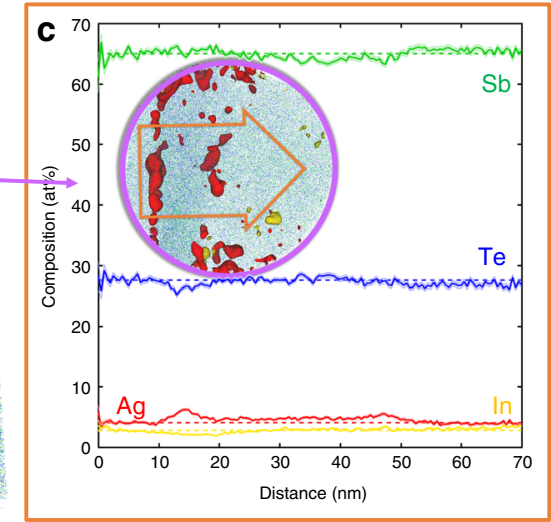

e



Fig. 3 Correlative APT-TEM investigation of grain boundary in crystalline AIST. a HAADF-STEM image of the needle-shaped AIST specimen along with the diffraction patterns from selected areas evidencing the high-angle grain boundary. b APT mapping of the AIST showing the segregation of Ag. c concentration profiles and ion count of the region of interest highlighted by blue cylinder. Proximity histogram concentration profiles of $\mathrm{Ag}$, In, $\mathrm{Sb}$, and $\mathrm{Te}$ for D: 7.5 at.\% Ag and E: 5.5 at.\% In iso-concentration surfaces. APT results show that $\sim 3.9$ at.\% Ag and $\sim 3.3$ at.\% In substitute the Sb in the quintuple layer, and excessive $\mathrm{Ag}$ segregates into the grain boundary

a

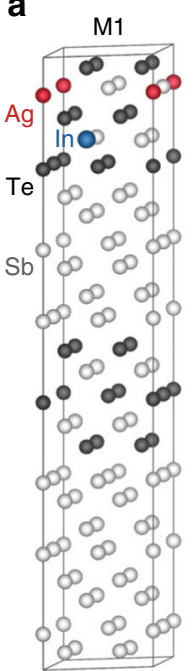

$E_{\text {tot }}=-401.18 \mathrm{eV}$

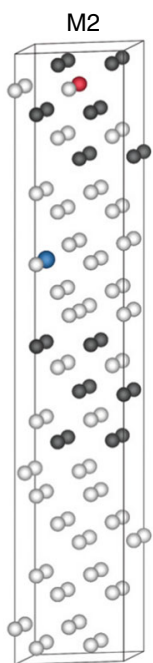

$-400.47 \mathrm{eV}$

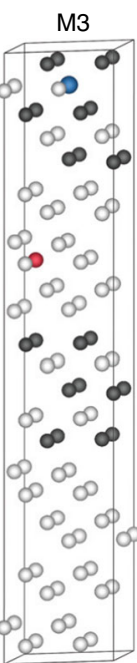

$-400.26 \mathrm{eV}$ b

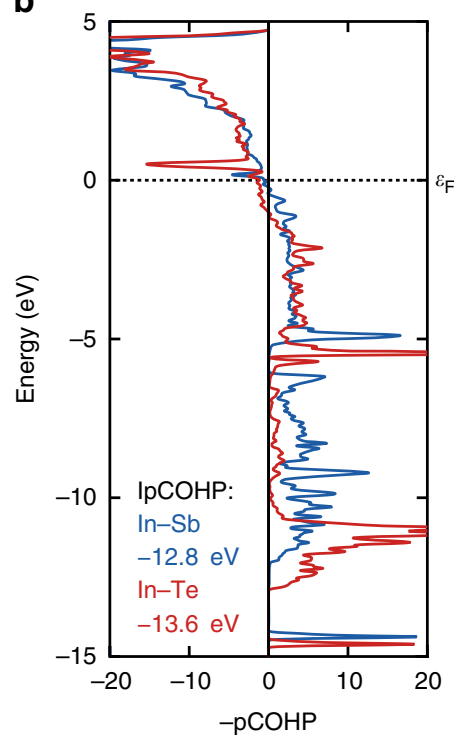

C

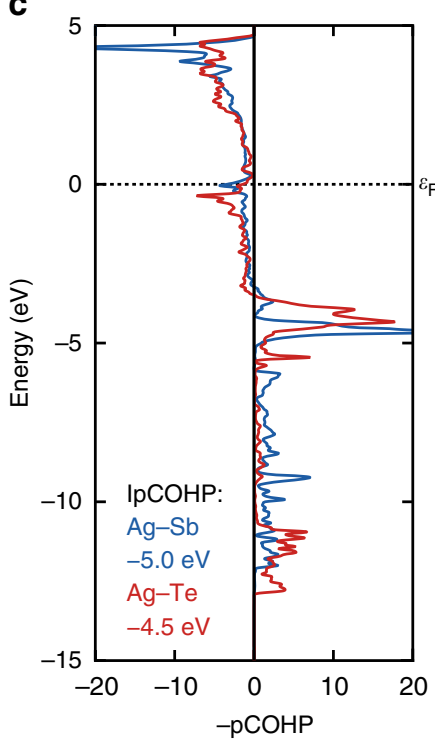

Fig. 4 Total energy and bonding analysis of AIST with different arrangements. a structural images of three typical AIST models (M1-3) including their total electronic energy. b projected COHP ( $p C O H P$ ) plots of $\mathrm{In}-\mathrm{Te}$ (red) and In-Sb (blue) interactions in models $\mathrm{M} 1$ and $\mathrm{M} 2$, respectively. c pCOHP plots of $\mathrm{Ag}-\mathrm{Te}$ (red) and $\mathrm{Ag}-\mathrm{Sb}$ (blue) interactions in models $\mathrm{M} 1$ and $\mathrm{M} 3$, respectively. The integral of the pCOHP up to the Fermi level (IpCOHP) is shown to better quantify the results. The energy axes are referenced to the Fermi level $\varepsilon_{F}$ 
Table 1 Mulliken charge analysis of the In and Ag-substituents in models M1-M3 as generated by LOBSTER

\begin{tabular}{|c|c|c|c|c|c|c|c|c|c|c|c|c|c|c|c|c|c|}
\hline \multicolumn{3}{|c|}{ In-Te (M3) } & \multicolumn{3}{|c|}{ In-Sb (M2) } & \multicolumn{3}{|c|}{ In-Te (M1) } & \multicolumn{3}{|c|}{$\mathrm{Ag}-\mathrm{Sb}(\mathrm{M} 3)$} & \multicolumn{3}{|c|}{$\mathrm{Ag}-\mathrm{Te}(\mathrm{M} 2)$} & \multicolumn{3}{|c|}{$\mathrm{Ag}-\mathrm{Te}$ (M1) } \\
\hline \multicolumn{2}{|c|}{ Atom } & \multirow{2}{*}{$\begin{array}{c}\text { Charge } \\
-0.09\end{array}$} & \multicolumn{2}{|c|}{ Atom } & \multirow{2}{*}{$\begin{array}{l}\text { Charge } \\
-0.19\end{array}$} & \multicolumn{2}{|c|}{ Atom } & \multirow{2}{*}{$\begin{array}{c}\text { Charge } \\
0.00\end{array}$} & \multicolumn{2}{|c|}{ Atom } & \multirow{2}{*}{$\begin{array}{c}\text { Charge } \\
0.39\end{array}$} & \multicolumn{2}{|c|}{ Atom } & \multirow{2}{*}{$\begin{array}{c}\text { Charge } \\
0.45\end{array}$} & \multicolumn{2}{|l|}{ Atom } & \multirow{2}{*}{$\begin{array}{r}\text { Charge } \\
0.45\end{array}$} \\
\hline 2 & In & & 2 & In & & 2 & In & & 1 & $\mathbf{A g}$ & & 1 & $\mathbf{A g}$ & & 1 & $\mathbf{A g}$ & \\
\hline 78 & $\mathrm{Te}$ & -0.10 & 16 & $\mathrm{Sb}$ & 0.01 & 80 & $\mathrm{Te}$ & -0.13 & 16 & $\mathrm{Sb}$ & -0.06 & 78 & $\mathrm{Te}$ & -0.24 & 78 & $\mathrm{Te}$ & -0.24 \\
\hline 84 & $\mathrm{Te}$ & -0.10 & 33 & $\mathrm{Sb}$ & 0.01 & 82 & $\mathrm{Te}$ & -0.27 & 33 & $\mathrm{Sb}$ & -0.13 & 84 & $\mathrm{Te}$ & -0.24 & 82 & $\mathrm{Te}$ & -0.27 \\
\hline 89 & $\mathrm{Te}$ & -0.20 & 34 & $\mathrm{Sb}$ & 0.01 & 86 & $\mathrm{Te}$ & -0.13 & 34 & $\mathrm{Sb}$ & -0.06 & 89 & $\mathrm{Te}$ & -0.30 & 90 & $\mathrm{Te}$ & -0.24 \\
\hline 96 & $\Sigma$ & -0.92 & & $\Sigma$ & 0.07 & & $\Sigma$ & -1.20 & & $\Sigma$ & -0.57 & & $\Sigma$ & -1.63 & & $\Sigma$ & -1.53 \\
\hline
\end{tabular}

cation, with charges between +0.39 and +0.45 . This comes as unexpected in Pauling's empirical electronegativities (In: 1.78, Ag: $1.93)^{31}$ but is well reflected in Mulliken's electronegativity scheme (In: 1.76, Ag: 1.47$)^{32,33}$ based on ionization energies and electron affinities.

This fundamental result of differing bonding character (practically neutral In: mostly covalent, but cationic Ag: electrostatic) suggest the interaction between the silver substituent and its first coordination shell are stabilized by ionic contributions, which is fully supported by the charge analysis of its first coordination sphere. A similar trend is found for Mulliken charge analysis of the four models using either single In or Ag atom, as show in Supplementary Table 1. These results illustrate that In substituents are more stable within the $\mathrm{Sb}_{2} \mathrm{Te}_{3}$ layer due to covalent contributions visible in the COHP bonding analysis. Ag atoms, on the other hand, are stabilized through ionic interactions in the immediate surrounding of the dopant. Therefore, both substituents are more stable between Te layers, but for different reasons: indium through covalent interactions and silver through ionic interactions.

Amorphous structure. After analyzing the atomic arrangement in AIST and its underlying bonding rationale, we now determine the precise roles of $\mathrm{Ag}$ and In in the AIST crystallization as mirrored from DFT-MD (molecular dynamics) simulations. We first analyze the change of amorphous structural features when incorporating $\mathrm{Ag}$ or In into a given $\mathrm{Sb}-\mathrm{Te}$ matrix. To uncover the individual role of $\mathrm{Ag}$ and In, systems with isolated $\mathrm{Ag}$, $\mathrm{Ag}_{8} \mathrm{Sb}_{128} \mathrm{Te}_{48}$ (ASI), as well as In dopants, $\mathrm{In}_{8} \mathrm{Sb}_{128} \mathrm{Te}_{48}$ (IST), are investigated. Structural characteristics of pure $\mathrm{Sb}_{128} \mathrm{Te}_{48}$ (ST) are presented as a reference. Their structures are obtained in two steps: first, the crystal structures, comprising of Sb bilayers and $\mathrm{Sb}_{2} \mathrm{Te}_{3}$ quintuple layers, where $\mathrm{Sb}$ is replaced by substituents, are melted at $3000 \mathrm{~K}$ for $50 \mathrm{ps}$; second, the high-temperature structures are relaxed at $600 \mathrm{~K}$ for $100 \mathrm{ps}$, where the last $60 \mathrm{ps}$ are used for structural feature analysis.

Four atomic structures of the last frames at $600 \mathrm{~K}$ are shown in Fig. 5a. Upon melting, the ST model has lost all features of the layered crystal structure. Te, however, is almost exclusively surrounded by $\mathrm{Sb}$, only very rarely by $\mathrm{Te}$, which is confirmed by just $1.3 \%$ of homopolar $\mathrm{Te}-\mathrm{Te}$ bonds found from the pair distribution function (PDF) in Fig. 5b. When adding $~ 4$ at.\% Ag to the ST model, the Ag atoms are located at the two sides of the amorphous AST structure, and none of them are found in the center. Moreover, Ag prefer to form clusters within amorphous AST (Fig. 5a), resulting in a strong first peak of Ag-Ag pairs near $3 \AA$ (Fig. 5b). This configuration supports the phase segregation upon crystallization found by X-ray diffraction ${ }^{34}$. In contrast, indium in IST disperses homogenously in the matrix, and few InIn bond are observed. This is supported by the very low first peak of an In-In pair near $4.0 \AA$ in its PDF curve. Interestingly, the Ag clusters, observed in AST, completely disappear after adding In. In contrast to the homogenous distribution of indium in IST, In atoms are now located in the two ends of the amorphous AIST. As a result, the intensity of the first peak for Ag-Te pairs in AIST are even higher than in AST, whereas fewer Ag-Sb pairs are observed. The calculated PDF of the amorphous AIST is in good agreement with the experimental data obtained from electron diffraction (Supplementary Fig. 7), implying that the amorphous structure obtained from MD simulation accurately represents the experimentally observed amorphous phase. Thus, adding In prevents $\mathrm{Ag}$ atoms from the formation of $\mathrm{Ag}$ cluster. In other words, Ag/In atoms are highly localized around Te in amorphous AIST, with Sb filling up the remaining Te coordination sphere, as is observed in the crystalline phase. Note that the distribution of Te atoms is barely altered after incorporating Ag and In, which can be seen from quite similar PDF curves of $\mathrm{Sb}-\mathrm{Te} / \mathrm{Te}-\mathrm{Te}$ pairs in ST and AIST. From the mean-square displacements (MSD) in Fig. $5 c$, we find that the diffusion coefficients $\left(D_{\mathrm{Ag}}\right)$ of $\mathrm{Ag}(3.61 \times$ $\left.10^{-10} \mathrm{~m}^{2} \mathrm{~s}^{-1}\right)$ and In $\left(2.36 \times 10^{-10} \mathrm{~m}^{2} \mathrm{~s}^{-1}\right)$ atoms in AIST are half of that of the systems with isolated $\mathrm{Ag}\left(7.31 \times 10^{-10} \mathrm{~m}^{2} \mathrm{~s}^{-1}\right)$ or In $\left(3.82 \times 10^{-10} \mathrm{~m}^{2} \mathrm{~s}^{-1}\right)$ substituents. This further proves that $\mathrm{Ag}$ and In atoms are mutually constrained in the AIST model. It is worthwhile to note that significantly reducing $D$ would increase the activation energy $E_{a}$ of amorphous phase $\left(D \propto 1 / E_{a}\right)^{16}$. Thus, both $\mathrm{Ag}$ and In play crucial roles in the stability of amorphous AIST, leading to its higher crystallization temperature compared to $\mathrm{ST}^{35-37}$. For all systems, the Te atoms possess the lowest $D_{\mathrm{Te}}$ and diffuse less compared to the other elements, which plays an important role in stabilizing the network structure. The structural and kinetic features of AIST clearly suggest that the two basic units in the crystalline phase, $\mathrm{Ag} / \mathrm{In}$ and Sb-enriched areas, can keep their basic frameworks against thermal fluctuations at $600 \mathrm{~K}$.

Molecular-dynamics simulations of recrystallization. Following the above, determining how the Ag/In or Sb-enriched regions influence the crystallization is of interest. Since AIST is a growthdominated PCM, here, we focus on the growth process of amorphous AIST. The structural evolution of amorphous AIST on annealing at $600 \mathrm{~K}$ were simulated using $\mathrm{MD}$, as shown in Fig. 6a to $\mathrm{f}$. To introduce the nucleus in this process, 45 atoms were fixed at the beginning, including $5 \mathrm{Ag}, 5 \mathrm{In}, 8 \mathrm{Sb}$, and $27 \mathrm{Te}$ atoms. Once the crystalline precursors consisting of $\mathrm{Ag} / \mathrm{In}-\mathrm{Te}$ cubes (Fig. 6a) are formed, the precursors first grow along adjacent In atoms in the quintuple layers within the next 20 ps, which is therefore an incubation period for the crystallization. After this period, a clear amorphous-crystalline interface can be observed. The crystalline-amorphous rim grows toward the amorphous area, forming ordered bilayer structures, that is, aligning the Sbcentered octahedra along the $c$ axes of the quintuple layer ${ }^{12}$ 
a

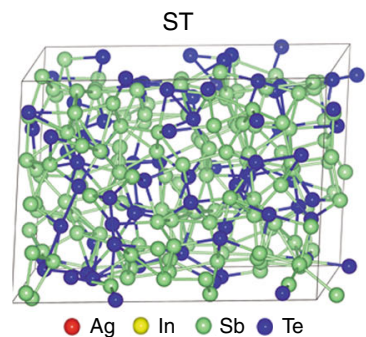

b
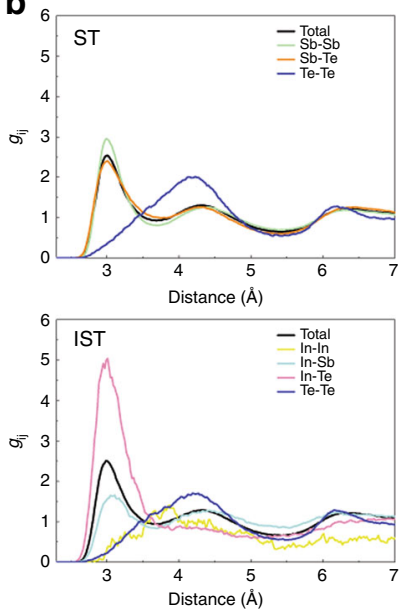
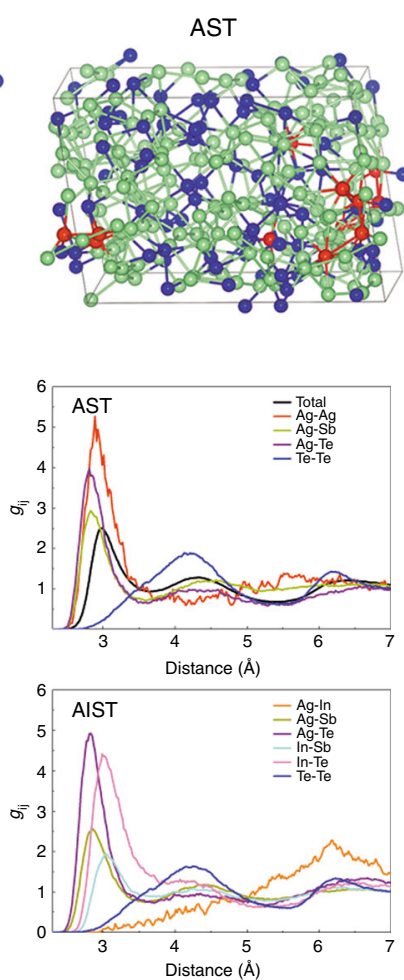

IST

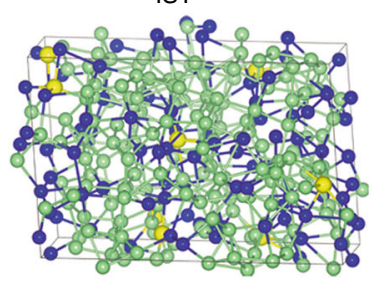

C
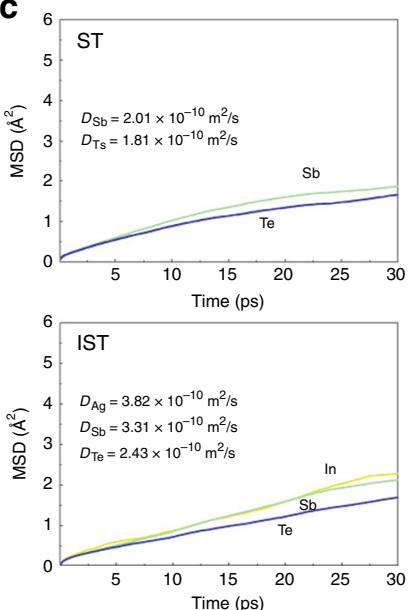
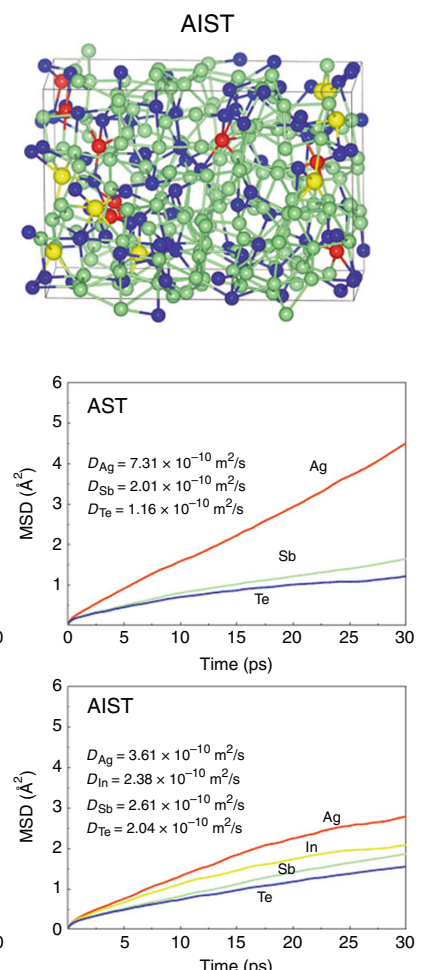

Fig. 5 The structural and kinetic features of $\mathrm{Sb}_{128} T e_{48}, \mathrm{Ag}_{8} \mathrm{Sb}_{128} T e_{48}, \mathrm{In}_{8} \mathrm{Sb}_{128} T e_{48}$, and $\mathrm{Ag}_{8} \mathrm{In}_{8} \mathrm{Sb}_{128} T \mathrm{e}_{48}$ systems at $600 \mathrm{~K}$. a snapshots of the last frames of these systems relaxed at $600 \mathrm{~K}$. b partial PDFs of these systems. $\mathbf{c}$ mean-square displacement (MSD) of these systems
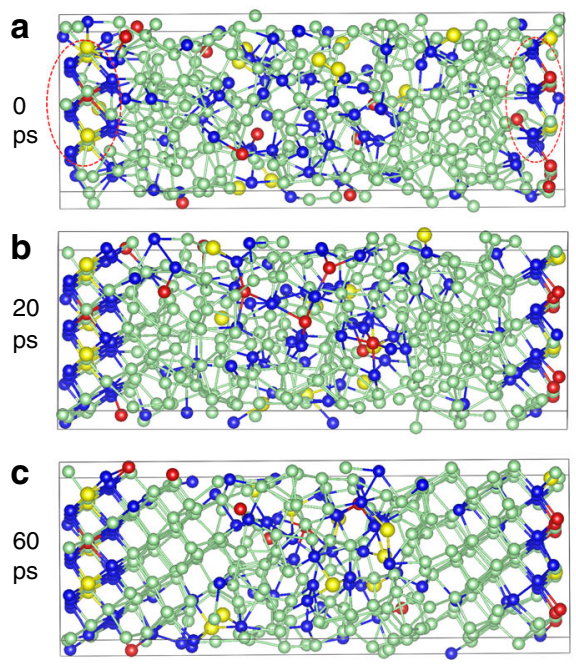

d
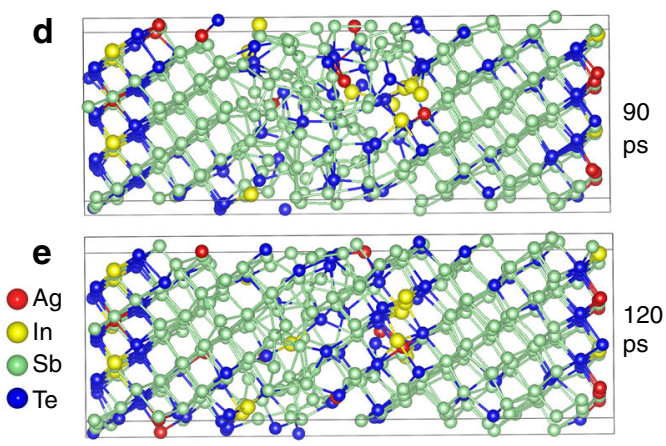

f

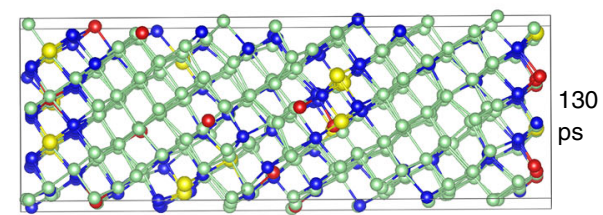

Fig. 6 Structural evolutions of the crystallization process in AIST. a-f snapshots of the crystallization process of AIST at 0, 20,60, 90, 120, and 130 ps ( $T=$ $600 \mathrm{~K}$ ). Red circles highlight the introduced crystalline precursor made of $\mathrm{Ag} / \mathrm{In}$ - $\mathrm{Te}$ cubes (45 atoms)

(Fig. 6b, c). This behavior shows the growth-dominated crystallization mechanism, a substantial difference from the nucleationdominated mechanism found in GST. As presented in Fig. $6 c$, the right bilayers (4 layers/60 ps) grow faster than the left ones (3 layers $/ 60 \mathrm{ps}$ ) with three $\mathrm{Ag}$ atoms. Compared to that, only $\sim 30 \mathrm{ps}$ are required to re-order the In-centered quintuple layer in the center (transition between Fig. 6d, e), while $\sim 90$ ps are necessary for the reconfiguration of three bilayers (Fig. 6a through $\mathrm{d}$ ). This indicates a slight reduction in growth speed through $\mathrm{Ag}$, whereas In accelerates crystallization. After $130 \mathrm{ps}$, an almost fully crystallized structure can be found, as shown in Fig. $6 \mathrm{f}$. The vast majority of $\mathrm{Ag}$ and In atoms are located in the quintuple layer, quite closely resembling the observed crystalline structures in
Fig. 2 and Supplementary Fig. 3. Subsequent short-order atom diffusion processes would occur to achieve the energetically favorable crystalline structure.

\section{Discussion}

The $~ 3.9$ at.\% Ag, through forming unexpectedly ionic $\mathrm{Ag}-\mathrm{Te}$ bonds, mutually constrain the random diffusion of the atoms in the Ag/In region. Both the ionic Ag-Te bonds and the mutually constrained effect are responsible for the higher crystallization temperature of AIST compared to ST. About 3.3 at.\% In atoms accelerate the reconfiguration of quintuple layers, speeding up the crystallization process, which in turn results in the faster 
a

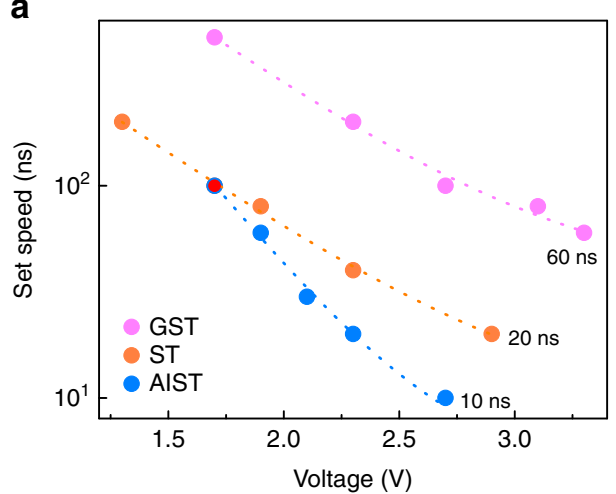

b

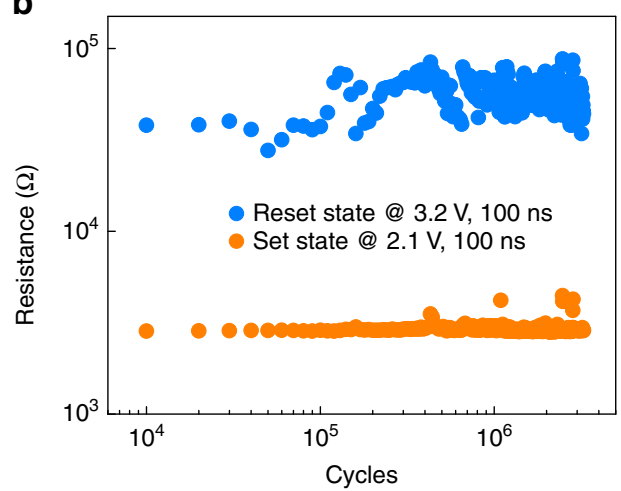

Fig. 7 Device performance of optimized AIST $\left(\mathrm{Ag}_{2.31} \mathrm{ln}_{4.56} \mathrm{Sb}_{61.27} T e_{31.86}\right)$ device. a set operation speed of GST, ST and optimized AIST-based devices. Optimized AIST presents the highest operation speed, $10 \mathrm{~ns}$, comparable to DRAM. b endurance performance of optimized AIST device. The cells can be repeatedly operated over 2 million times

operation speed of AIST. To sum up, In and Ag in AIST interact to stabilize the amorphous phase, while In atoms speed up crystallization. After forming the Ag/In-enriched quintuple layers, the growth process is subsequently achieved via aligning the $\mathrm{Sb}$-centered octahedra in Sb-enriched regions along the $c$ axes of the quintuple layer, resulting in the growth-dominated crystallization behavior.

According to the aforementioned results, it is reasonable to lower the concentration of Ag in AIST to avoid possible phase segregation. In fact, AIST with 2.3 at. $\%$ Ag concentration already possesses sufficient stability against crystallization (at $173{ }^{\circ} \mathrm{C}$ ). Device cells using the optimized AIST film show the transformation into a low-resistance state (crystallization state) in just $10 \mathrm{~ns}$ (Fig. 7a), comparable to DRAM. The speed is twice as fast as the ST-based cell, and six times faster than a GST-based one (see detailed information in Supplementary Fig. 8). The shortened switching process is achieved by acceleration of the crystallization process through In dopant engineering. Moreover, a reduced Ag content indeed prolongs the device lifetime of the cell, over three million cycles, as shown in Fig. 7b, one order of magnitude longer than an AIST-based cell ( $\sim 10^{5}$ cycles).

We have now quantitatively determined the distribution of atomic-scale dopants in PCMs by advanced experimental and also theoretical techniques. Transferring the structure obtained into DFT calculations, the precise role of each element during crystallization was described and modelled. This accomplishment has been enabled by a detailed understanding of the crystallization behavior of Ag-In-Sb-Te alloys, in particular the role of small amounts of Ag and In. We thus hope that our work serves as a rational signpost for boosting phase-change memory through dopant engineering.

\section{Methods}

Film deposition and stoichiometry characterization. Six hundred nanometer thick AIST films were sputtered by alloy targets. The compositions of these films were identified by atom-probe tomography and arrived at Ag:In:Sb:Te = 5.13:3.32:65.60:25.93 in at.\%.

Cs-corrected TEM characterization. Amorphous AIST film was cut into TEM lamella using a dual-beam focused ion beam (FIB, FEI Helios Nanolab 600). The atomic-resolution STEM experiment was performed on a JEOL JEM-ARM300F microscopy equipped with a cold field-emission electron gun, dual-probe Cs-corrector, three detectors as well as an energy-dispersive X-ray (EDX) spectroscope. In situ like STEM investigations were performed to study the structural evolution of crystalline AIST. The investigation temperature ranges from room temperature to $350^{\circ} \mathrm{C}$. The sample was heated by TEM heating holder with a heating rate of $10^{\circ} \mathrm{C} /$ min, which stopped at $200,250,300$, and $350^{\circ} \mathrm{C}$, respectively. To get the atomresolved mapping, this sample needed to be put into the EDX holder. Another sample was annealed by long-time annealing process, $300^{\circ} \mathrm{C}$ for $30 \mathrm{~min}$, and then investigated by Cs-corrected TEM to check the influence of annealing time on the atomic arrangement of crystalline AIST. The highest resolution of the Cs-corrected TEM, $0.63 \AA$, has been reported. The high-angle annular dark-field (HAADF) images were obtained at $80 \mathrm{kV}$ or $300 \mathrm{kV}$, while EDX mappings were carried out at $80 \mathrm{kV}$. The size of EDX mapping were $1024 \times 1024$ pixels. The probe current was $\sim 32 \mathrm{pA}$. The acquisition time was $30-80 \mathrm{~min}$.

To get the experimental structure information of the amorphous AIST, we extracted the pair distribution function (PDF) from the electron diffraction data of the amorphous AIST film. The electron diffraction pattern was obtained using JEOL JEM-ARM300F microscopy. The experimental data reduction techniques that used to obtain PDF can be found in refs. ${ }^{38,39}$.

Atom-probe tomography analysis. Six needled-sharped tips of crystalline AIST were prepared using the standard lift-out procedure by FIB, with a diameter of the apex less than $100 \mathrm{~nm}$. These tips were mounted on a half-cut molybdenum TEM grid, which were then analyzed by JEOL JEM-2100F TEM in bright field mode as well as HAADF mode. The EDX mapping of these tips were also obtained. Subsequently, these tips were put into a $3 \times 10^{-11} \mathrm{mbar}$ high vacuum in local electrode atom-probe (LEAP 5000 XR, Cameca Instruments). Applied a DC voltage of 2-6.5 $\mathrm{kV}$ and illuminated by $10 \mathrm{ps}$ laser pulses in the atom-probe tomography, surfaces atoms of the needle-shaped sample were ionized, filed evaporated and subsequently projected onto a position-sensitive detector ${ }^{24}$. The $x$ - and $y$-coordinates of the ions registered by the detector, as well as the calculated $z$-coordinate were used to reconstruct the 3D map. Moreover, these ions can be chemically identified based on time-of-flight mass spectrometry. Reconstruction and analysis of 3D maps were carried out using software IVAS 3.6.14.

Device preparation and measurement. One hundred nanometer thick PCM film was deposited on the device with a bottom electrode diameter of $190 \mathrm{~nm}$. Ten nanometer thick TiN and 300-nm-thick Al films are subsequently deposited on the PCM film, severed as the top electrode. The device performances were characterized by a parameter analyzer (Keithley $2400 \mathrm{C}$ ) and a pulse generator (Tektronix AWG5200B).

Bond and charge computational details. Density-functional theory (DFT) calculations employed the 'D 3 ' van der Waals correction ${ }^{40}$ on top of the PBE functional ${ }^{41}$. Plane-wave basis sets and the projector augmented wave (PAW) method $^{42}$ were used as implemented in the Vienna Ab Initio Simulation Package $(\text { VASP })^{43-45}$. The energy cutoff for the plane-wave expansion was set to $500 \mathrm{eV}$ with an electronic convergence criterion of $10^{-7} \mathrm{eV}$. Structural optimization was performed until residual forces fell below $5 \times 10^{-3} \mathrm{eV}^{-1}$ and reciprocal space was sampled on $\Gamma$-centered k-point grids with densities between 0.02 and $0.04 \AA^{-1}$. Chemical-bonding analyses of plane-wave data, as well as Mulliken charge analysis, were performed using LOBSTER $^{27-29,46}$.

Molecular-dynamics simulations. Molecular-dynamics simulations were carried out based within the framework of density-functional theory (DFT-MD). The Kohn-Sham equations were solved using the VASP package and the $\Gamma$ zone center only. The valence electron and core interactions were described using the projector augmented wave (PAW) method, where the valence electrons are $4 d^{10} 5 s^{1}$ for $\mathrm{Ag}$, $4 d^{10} 5 s^{2} 5 p^{1}$ for In, $5 s^{2} 5 p^{3}$ for $\mathrm{Sb}$, and $5 s^{2} 5 p^{4}$ for Te. Electron exchange and correlation were treated as described above with a kinetic energy cutoff of $250 \mathrm{eV}^{40,41,47}$. The convergence criterion was $2 \times 10^{-5} \mathrm{eV}$ for electronic convergence and $0.2 \mathrm{eV} / \AA$ for force. Models were first melted at $3000 \mathrm{~K}$ for $50 \mathrm{ps}$ with a time step $\delta t=2 \mathrm{fs}$, and then they were relaxed at $600 \mathrm{~K}$ for $100 \mathrm{ps}$. In the simulations, the ParrinelloRahman (NpT) dynamics with Langevin thermostat was used. The recrystallization 
simulation model (384 atoms, including $16 \mathrm{Ag}, 16 \mathrm{In}, 256 \mathrm{Sb}$, and $96 \mathrm{Te}$ atoms) with periodic boundary conditions was acquired as follows: 45 atoms, including 5 $\mathrm{Ag}, 5 \mathrm{In}, 8 \mathrm{Sb}$, and $27 \mathrm{Te}$ atoms, were fixed during melting and growing processes. The unfixed atoms were maintained at $3000 \mathrm{~K}$ for $15 \mathrm{ps}$ to erase the information of crystalline state. The crystal-growth simulation for AIST was performed at $600 \mathrm{~K}$. The total simulation time was 130 ps. The energy cutoff was $250 \mathrm{eV}$ and the time step for the simulations was $3 \mathrm{fs}$.

Mean-square displacement and Einstein relationship. The mean-square displacement is defined as

$$
\operatorname{MSD}_{t}=\frac{1}{N} \sum_{i=1}^{N}\left[x_{i}\left(t+t_{0}\right)-x_{i}\left(t_{0}\right)\right]^{2}
$$

where $N$ is the total number of atoms, $x_{i}\left(t_{0}\right)$ is the reference position of the $i^{\text {th }}$ atom at time $t_{0}, x_{i}\left(t+t_{0}\right)$ is the position of the $i^{\text {th }}$ atom at time $\left(t+t_{0}\right)$.

The diffusion coefficient $D$ is determined according to the Einstein relationship

$$
\mathrm{MSD}_{t}=2 D \cdot t
$$

where $t$ is the time of atom movement.

\section{Data availability}

The data that support the findings of this study are available from the corresponding author upon reasonable request.

Received: 4 December 2018 Accepted: 15 July 2019

Published online: 06 August 2019

\section{References}

1. Atwood, G. Phase-change materials for electric memories. Science 321, 210-211 (2008).

2. Burr, G. W. et al. Phase change memory technology. J. Vac. Sci. Technol. B 28, 223-261 (2010).

3. Burr., G. W. et al. Overview of candidate device technologies for storage-class memory. IBM J. Res. Dev. 52, 449-463 (2008).

4. https://www.anandtech.com/show/9541/intel-announces-optane-storagebrand-for-3d-xpoint-products.

5. Wuttig, M. \& Yamada, N. Phase-change materials for rewriteable data storage. Nat. Mater. 6, 824-832 (2007).

6. Wuttig, M. et al. Incipient metals: functional materials with a unique bonding mechanism. Adv. Mater. 30, 1803777 (2018).

7. Kau D. et al. A stackable cross point phase change memory. in Proc. Int. Electron Device Meeting (IEDM) Tech. Dig. (Baltimore, MD, USA, 2009), pp. 1-4. https://ieeexplore.ieee.org/abstract/document/5424263.

8. Czubatyj, W., Lowrey T., Kostylev, S. Current reduction in ovonic memory devices. In E/PCOS 2006, 143-152 (Grenoble, France, 2006).

9. Iwasaki, H., Ide, Y., Harigaya, M., Kageyama, Y. \& Fujimura, I. Completely erasable phase change optical disk. Jpn. J. Appl. Phys. 31, 461-465 (1992).

10. Iwasaki, H. et al. Completely erasable phase change optical disc II: application of Ag-In-Sb-Te mixed-phase system for rewritable compact disc compatible with CD-velocity and double CD-velocity. Jpn. J. Appl. Phys. 32, 5241-5247 (1993).

11. Zhou, G. F. Materials aspects in phase change optical recording. Mater. Sci. Eng. A 304, 73-80 (2001).

12. Matsunaga, T. et al. From local structure to nanosecond recrystallization dynamics in AgInSbTe phase-change materials. Nat. Mater. 10, 129-134 (2011).

13. Matsunaga, T., Umetani, Y. \& Yamada, N. Structural study of a $\mathrm{Ag}_{3.4} \mathrm{In}_{3.7} \mathrm{Sb}_{76.4} \mathrm{Te} 16.5$ quadruple compound utilized for phase-change optical disks. Phy. Rev. B 64, 184116 (2001).

14. Raoux, S., Sweet, J. L. J. \& Kellock, A. J. Crystallization properties of ultrathin phase change films. J. Appl. Phys. 103, 114310 (2008).

15. Lee, B.-S. et al. Observation of the role of subcritical nuclei in crystallization of a glassy solid. Science 326, 980-984 (2009).

16. Zhang, W. et al. How fragility makes phase-change data storage robust: insights from ab initio simulations. Sci. Rep. 4, 6529 (2014).

17. Duggin, M. J. A high-pressure phase in arsenic and its relation to pressureinduced phase changes in group 5B elements. J. Phys. Chem. Solids 33, 1267-1271 (1972).

18. Gaspard, J.-P., Pellegatti, A., Marinelli, F. \& Bichara, C. Peierls instabilities in covalent structures I. Electronic structure, cohesion and the $Z=8-N$ rule. Philos. Magaz. B 77, 727-744 (1998).

19. Kifune, K. et al. Crystal structures of X-phase in the Sb-Te binary alloy system. Cryst. Res. Technol. 48, 1011-1021 (2013).
20. Küpers, M. et al. Unexpected Ge-Ge contacts in the two-dimensional $\mathrm{Ge}_{4} \mathrm{Se}_{3} \mathrm{Te}$ phase and analysis of their chemical cause with the density of energy (DOE) function. Angew. Chem. Int. Ed. 56, 10204-10208 (2017).

21. Zhu, M. et al. One order of magnitude faster phase change at reduced power in Ti-Sb-Te. Nat. Commun. 5, 4086 (2014).

22. Zhu, M. Ti-Sb-Te Phase Change Materials Component optimisation, Mechanism and Applications (Springer, Singapore, 2017).

23. Gault, B. Atom Probe microscopy (Springer, 2012).

24. Zhu, M. et al. Unique bond breaking in crystalline phase change materials. Adv. Mater. 30, 1706735 (2018).

25. Hellman, O. C., Vandenbroucke, J. A., Rusing, J., Isheim, D. \& Seidman, D. N Analysis of three-dimensional atom-probe data by the proximity histogram. Microsc. Microanal. 6, 437-444 (2000).

26. Chou, C.-C., Hung, F.-Y. \& Lui, T.-S. Role of crystallized phases in sheet resistance of amorphous AgInSbTe chalcogenide film. Scr. Mater. 56, 1107-1110 (2007)

27. Dronskowski, R. \& Blöchl, P. E. Crystal orbital Hamilton populations (COHP): energy-resolved visualization of chemical bonding in solids based on density-functional calculations. J. Phys. Chem. 33, 8617-8624 (1993).

28. Maintz, S., Deringer, V. L., Tchougréeff, A. L. \& Dronskowski, R. Analytic projection from plane-wave and PAW wavefunctions and application to chemical-bonding analysis in solids. J. Comput. Chem. 34, 2557-2567 (2013).

29. Maintz, S., Deringer, V. L., Tchougréeff, A. L. \& Dronskowski, R. Loster: a too to extract chemical bonding from plane-wave based DFT. J. Comput. Chem. 37, 1030 (2016)

30. Li, W.-L., Ertural, C., Bogdanovski, D., Li, J. \& Dronskowski, R. Chemical bonding of crystalline $\mathrm{LnB}_{6}(\mathrm{Ln}=\mathrm{La}-\mathrm{Lu})$ and Its relationship with $\mathrm{Ln}_{2} \mathrm{~B}_{8}$ gasphase complexes. Inorg. Chem. 57, 12999-13008 (2018).

31. Allred, A. L. Electronegativity values from thermochemical data. J. Ionrg. Nucl. Chem. 17, 215-221 (1961).

32. Mulliken, R. S. A new electroaffinity scale; together with data on valence states and on valence ionization potentials and electron affinities. J. Chem. Phys. 2, 782-793 (1934)

33. Mulliken, R. S. Electronic structures of polyatomic molecules and valence VI. On the method of molecular orbitals. J. Chem. Phys. 3, 375-378 (1935).

34. Chen, Y.-M. \& Kuo, P. C. Effect of Ag or Cu doping on erasable phase-change Sb-Te thin films. IEEE Trans. Mag. 34, 432-434 (1998)

35. Lankhorst, M. H. R., Pieterson, L. V., Schijndel, M. V., Jacobs, B. A. \& Rijpers, J. C. N. Prospects of doped Sb-Te phase-change materials for high-speed recording. Jpn. J. Appl. Phys. 42, 863-868 (2003).

36. Lee, M. L., Shi, L. P., Tian, Y. T., Gan, C. L. \& Miao, X. S. Crystallization behavior of $\mathrm{Sb}_{70} \mathrm{Te} 30$ and $\mathrm{Ag}_{3} \mathrm{In}_{5} \mathrm{Sb}_{60} \mathrm{Te}_{32}$ chalcogenide materials for optical media applications. Phys. Stat. Sol. (a) 205, 340-344 (2008).

37. Matsushita, T., Suzuki, A., Nishiguchi, T., Shibata, K. \& Okuda, M. Phasechange optical recording films with $\mathrm{AgInTe}_{2}$-Sb-Te system. Jpn. J. Appl. Phys. 34, 519-520 (1995).

38. Mitchell, D. R. G. \& Petersen, T. C. RDFTools: a software tool for quantifying short-range ordering in amorphous materials. Microsc. Res. Tech. 75, 153-163 (2012).

39. Zhu, M. et al. Understanding the crystallization behavior of the as-deposited Ti-Sb-Te alloys through real-time radial distribution functions. Nanoscale 7, 9935-9944 (2015).

40. Grimme, S., Antony, J., Ehrlich, S. \& Krieg, H. A consistent and accurate ab initio parametrization of density functional dispersion correction (DFT-D) for the 94 elements H-Pu. J. Chem. Phys. 132, 154104 (2010).

41. Perdew, J. P., Burke, K. \& Ernzerhof, M. Generalized gradient approximation made simple. Phys. Rev. Lett. 77, 3865-3868 (1996).

42. Blöchl, P. E. Projector augmented-wave method. Phys. Rev. B 50, 17953-17979 (1994).

43. Kresse, G. \& Furthmüller, J. Efficient iterative schemes for ab initio totalenergy calculations using a planed-wave basis set. Phys. Rev. B 54, 11169-11186 (1996).

44. Kresse, G. \& Furthmüller, J. Efficient ab-initio total energy calculations for metals and semiconductors using a plane-wave basis set. Comput. Mater. Sci. 6, 15-50 (1996)

45. Kresse, G. \& Joubert, D. From ultrasoft pseudopotentials to the projector augmented-wave method. Phys. Rev. B 59, 1785-1775 (1999).

46. Deringer, V. L., Tchougréeff, A. L. \& Dronskowski, R. Crystal orbital Hamilton population (COHP) analysis as projected from plane-wave basis sets. J. Phys. Chem. A 115, 5461-5466 (2011).

47. Kresse, G. \& Hafner, J. Ab initio molecular dynamics for liquid metals. Phys. Rev. B 47, 558-561 (1993).

\section{Acknowledgements}

Financial support was provided by the National Key Research and Development Program of China (2017YFB0206101), Strategic Priority Research Program of the Chinese Academy of Sciences (XDPB12) and the National Natural Science Foundation of China (61504157). M.Z. acknowledges support by the Hundred Talents Program (Chinese 
Academy of Sciences) and the Shanghai Pujiang Talent Program (18PJ1411100). P.M.K, R.D. and M.W. acknowledge support by the Deutsche Forschungsgemeinschaft (SFB 917) and the Jülich-Aachen Research Alliance (JARA-HPC project jara0033).

\section{Author contributions}

M.Z., X.C. and S.L. prepared the specimens and carried out the TEM experiments. M.Z., B.G. and S.L. carried out the APT experiments and analyzed the APT results. P.M.K. and R.D. performed the COHP calculations. W.S. performed the amorphous structure simulation. T.L. and M.Z. performed the recrystallization simulation. J.S and M.Z. prepared the device and measured the performances. M.Z., P.M.K, B.G., M.W. and R.D. wrote this paper. The project was initiated and conceptualized by M.Z. and Z.S.

\section{Additional information}

Supplementary Information accompanies this paper at https://doi.org/10.1038/s41467019-11506-0.

Competing interests: The authors declare no competing interests.

Reprints and permission information is available online at http://npg.nature.com/ reprintsandpermissions/
Peer review information: Nature Communications thanks the anonymous reviewer(s) for their contribution to the peer review of this work.

Publisher's note: Springer Nature remains neutral with regard to jurisdictional claims in published maps and institutional affiliations.

(c) (i) Open Access This article is licensed under a Creative Commons Attribution 4.0 International License, which permits use, sharing, adaptation, distribution and reproduction in any medium or format, as long as you give appropriate credit to the original author(s) and the source, provide a link to the Creative Commons license, and indicate if changes were made. The images or other third party material in this article are included in the article's Creative Commons license, unless indicated otherwise in a credit line to the material. If material is not included in the article's Creative Commons license and your intended use is not permitted by statutory regulation or exceeds the permitted use, you will need to obtain permission directly from the copyright holder. To view a copy of this license, visit http://creativecommons.org/ licenses/by/4.0/.

(C) The Author(s) 2019 